El Guiniguada • Revista de investigaciones y experiencias en Ciencias de la Educación

\title{
LA FORMACIÓN PERMANENTE DEL PROFESORADO: ANÁLISIS Y SENTIDO
}

\section{THE PERMANENT TEACHER TRAINING: ANALYSIS Y SENTIDO}

\author{
María Elena Díaz Negrín \\ Centro del Profesorado Las Palmas de Gran Canaria
}

Recibido: 05/04/2015/Aceptado: 19/04/2015

\section{RESUMEN}

Hemos entrado en una sociedad que exige de los profesionales de la educación una permanente actividad de formación y aprendizaje. Una formación que debe ir acorde con los cambios que esa sociedad está demandando. Unos cambios que deben llevar a replantear el trabajo de los profesores en el aula y la escuela, que conduzcan a una estructura escolar más flexible y adaptada a las posibilidades y necesidades individuales del alumnado. Para que estos cambios se gesten, la formación del profesorado debe estar diseñada para tal fin.

Palabras clave: formación permanente, necesidad, utilidad, satisfacción, innovación.

\section{ABSTRACT}

We have entered a society that requires a permanent activity of training of teaching professionals. Training must be consistent with the changes that society is demanding. A few changes that should lead to rethink the work of teachers in the classroom and the school, leading to a school structure more flexible and adapted to the individual needs of students and the possibilities. To make these changes is gesten, teacher training must be designed for this purpose.

Keywords: lifelong learning, need, usefulness, satisfaction, innovation. 


\section{INTRODUCCIÓN}

Es indiscutible que en muchos estudios, en foros diversos y en el seno de la administración educativa se ha dejado de manifiesto la importancia del papel del profesorado como elemento clave para lograr la calidad educativa. Por ello, el profesorado debe verse asistido en el desempeño de sus funciones (Tejada, 2001).

Una forma de cuidarlo es velar por su formación y actualización docente y dotarlo de recursos y estrategias que le permitan dar respuestas a su alumnado y a la sociedad actual. Pero ¿es necesaria la formación permanente del profesorado? ¿Es útil para su desarrollo profesional? ¿Se da respuesta a estas necesidades? ¿Qué se le ofrece? ¿Qué aspectos debe abordar esa formación?

Si la sociedad de la información y la comunicación vive cambios a un ritmo vertiginoso en todos los ámbitos y esto repercute directamente en la educación, en cada una de las escuelas, en sus aulas y en los discentes, el profesorado no puede mantenerse al margen y tiene que formarse para dar respuesta. Formarse, porque el Lifelong Learning se reconoce como un derecho al que tienen acceso todos los ciudadanos (Longworth, 2001 citado en García y Castro, 2012) y formarse porque a través de su reciclaje se le permite aprender nuevos contenidos, adquirir nuevas habilidades, manejar nuevas herramientas, conocer estrategias de enseñanza, conocer formas de trabajar en equipo y en red, investigar e implementar metodologías activas para preparar a sus estudiantes a vivir en esa sociedad de la información y comunicación.

Con estos cambios de los que hablamos, si el alumnado es distinto al de antes, los equipos docentes han de cambiar en el mismo sentido y adaptarse a las nuevas circunstancias. Para conseguirlo tienen a su disposición un potente recurso: los planes de formación permanente (Red Eurydice, 2006).

\section{BINOMIO NECESIDAD-UTILIDAD DE LA FORMACIÓN DEL PROFESORADO}

¿Somos conscientes de esa necesidad formativa del profesorado como clave para enseñar al alumnado a vivir en la sociedad actual, con las correspondientes exigencias? Cada día somos más conscientes de ello, porque hemos experimentado, a través de la vida del aula y del intercambio de reflexiones con colegas profesionales, que lo que antes nos valía con nuestro alumnado, ahora no. Pero además de plantear un tema de consciencia, coincidimos con Marcelo (2002) al considerar que para el profesorado el aprendizaje permanente no es una opción a elegir, sino una obligación moral para una profesión comprometida con el conocimiento. Y si vamos un poco más allá, podríamos decir que la formación permanente es la opción para la supervivencia del profesorado. Este siente que para dar respuestas a los objetivos que se plantea hoy en día la educación, como es paliar las tasas de abandono escolar, tiene que 
ir en busca de ese alumnado, conquistarlo, cuidar su ambiente de aprendizaje, vincular los aprendizajes con utilidades, con un sentido y un para qué. Este logro no es sencillo y requiere que el profesorado esté formado y avalado por su experiencia profesional.

Y si hablamos de necesidad, debemos reflexionar sobre la utilidad de la formación del profesorado. Este debe sentir que la formación que se le propone es una ayuda a su desarrollo profesional y que incide directamente en las situaciones que encuentra en su centro, que le enseña estrategias colaborativas (Gairín, 2008 citado en García y Castro, 2012), a partir de la participación; y que lo forma en metodologías diversas, que le permite el intercambio de experiencias, el debates, análisis y reflexión y que esto le facilita su desempeño. Pero para ello, Imbernón (2006, citado en García y Castro, 2012), es esencial activar el proceso de la evaluación de la formación y valorar si realmente tiene impacto en el desarrollo profesional y en la innovación de los centros. Este proceso es clave pero no profundizaremos en este aspecto, porque sería un análisis y reflexión para tratar en otro artículo, como punto único.

\section{Aspectos QUE DEBE CONTEMPLAR LA FORMACIÓN DEL PROFESORADO}

La enseñanza está organizada de manera que los docentes interpretan y ponen en práctica las normas educativas y el currículo. Todas las influencias sobre la calidad de la educación están mediadas por el docente y por su acción. Para bien o para mal, los profesores determinan la calidad de la educación, Clark (1995, citado en Grau, Gómez y Perandones, 2009) aunque es obvio que no es el único agente determinante. Pero vamos a ver cómo puede responder el profesorado a esa calidad a través de la formación.

\subsection{Formación en el contexto real}

Para que la respuesta formativa sea eficiente, Medina y Domínguez (1989, citado en Grau, Gómez y Perandones, 2009), es fundamental proporcionar al profesorado una formación centrada en su lugar de trabajo, donde se le capacite para analizar el sistema educativo y desarrollar su práctica como una labor de innovación. En la actualidad en Canarias se da respuesta a esta necesidad a través de los planes de formación en centros que permite contextualizar la formación acorde con el plan de mejora de cada centro, integrando en la rutina de trabajo la formación. Esta modalidad formativa da respuesta a la apuesta de Camargo, Calvo, Franco, Vergara, Londoño, Zapata, et al. (2004, citado en García y Castro, 2012) que consideran que las necesidades de formación han de estar originadas en las demandas del contexto de cada centro educativo. 


\subsection{Formación para la innovación}

La formación debe promover la actualización pedagógica, despertando y activando en el profesorado la capacidad de innovación, reflexión y crítica del trabajo diario, asumiendo con sus compañeros proyectos comunes, de tal forma que se sienta comprometido con el cambio. En la Comunidad Autónoma de Canarias la convocatoria de la Red de Centros Innovadores para el curso 2014-15 ha permitido potenciar esa innovación a través de la formación, facilitando espacios para la reflexión, análisis y encuentros entre el profesorado de distintas etapas educativas, a través de talleres con diversas temáticas: aprendizaje cooperativo, aprendizaje basado en proyectos, aprendizajes basados en destrezas de pensamiento,...Se ha nutrido así, su maleta de acciones didácticas para vincularla con la praxis educativa. Una praxis que reduce la tasa de abandono escolar, mejora los resultados académicos del alumnado, desarrolla aprendizajes competenciales y coordina el paso entre las etapas educativas, como indica la Resolución de la Dirección General de Ordenación, Innovación y Promoción Educativa por la que se relacionan los centros seleccionados para participar en la red de centros innovadores para la continuidad escolar (2014-2015).

Se trata de activar aquellas capacidades que ayuden a superar los factores que obstaculizan la renovación metodológica y pedagógica, como pueden ser, la falta de incentivos al reconocimiento de la labor docente, la falta de formación, la rutina, el miedo al cambio...y, por supuesto, conseguir con todo ello, un docente satisfecho de su práctica docente y de su interacción con el entorno laboral.

\subsection{Formación desde la reflexión-cooperación}

Para Pérez Gómez (2005, citado en Gómez, 2011) es esencial el proceso de acción y reflexión cooperativa, de indagación y experimentación, donde el profesor aprende al enseñar, y enseña, porque aprende. Haciéndonos eco del sentir de este autor la formación debe apoyar la figura del profesor como un sujeto reflexivo e innovador, cuya formación se desarrolle en el contexto de su trabajo, junto con el resto de sus compañeros, potenciando el trabajo cooperativo entre los profesores de una misma o distinta área o centro, como vía de la propia formación profesional. Esto hace que recobre importancia los espacios de coordinación entre colegas, espacios para el análisis de la situación del alumnado, para acordar el desarrollo de proyectos conjuntos, para compartir buenas prácticas educativas, para la evaluación de esas acciones y para diseñar un plan de acción consensuado. Solo si hacemos de la reflexión del profesorado una estrategia para su propia formación, se experimentarán avances. 


\subsection{Formación autónoma del profesorado}

Debemos contemplar también que hay profesorado que decide aprender por sí mismo para su mejora personal o profesional, diseñando su itinerario formativo. Los cursos a distancia, que profundizan sobre una determinada temática, y los seminarios permanentes dan respuesta a esta modalidad de gestionar la formación. Hoy en día, los seminarios intercentros, organizados por la Red de Centros del Profesorado, permite el encuentro entre profesorado con diferentes niveles de formación, que intercambian experiencias y reflexionan sobre la práctica educativa. Como señala Juan Manuel Esteve (2009, citado en Tiana, 2011), entre las actividades formativas que mejor ayudan al docente están las que se basan en el trabajo cooperativo de grupos de profesores de la misma materia que disponen de un espacio para compartir sus problemas en las aulas, intercambiar y elaborar nuevos materiales, compartir sus estrategias didácticas y reflexionar sobre los desafíos y las nuevas soluciones. Otra línea alternativa es el campus de autoformación que permite la adquisición de aprendizajes de manera autónoma.

Serían este tipo de actividades las que complementarían a muchos cursos, conferencias y jornadas de más corto alcance (Escudero, 2009b). Cursos de temática diversa (TIC, hablar otra lengua, convivencia,...) y de teleformación.

\subsection{Formación para liderar}

Todo proceso que se quiera poner en práctica, de la índole que sea, requiere de líderes que inspiren. La formación del líder debe facilitar estrategias para consolidar, defender y difundir el perfil cultural de la entidad educativa que representa para que sea asumida por todos sus miembros (Gento, S., 2002: 203). El informe de la OECD (Organización para la Cooperación y el Desarrollo Económicos) sobre Mejora del Liderazgo en las Instituciones Escolares, en su volumen 2 se refiere a la necesidad de redefinir las responsabilidades del liderazgo en las instituciones educativas. En esa redefinición, debe tenerse en cuenta que gestionar un cambio educativo, supone una alteración en el diseño organizativo, estrategia, métodos o cualquier otro intento de influir en los miembros de una organización para promover los cambios necesarios. Lograr este reto es un proceso complejo y la formación debe ayudar a los líderes a proyectar su visión para ganar apoyos para ella, a ser consistentes, persistentes y mantener el ánimo de la organización para empoderar a aquellos que deben asumir alguna responsabilidad en el movimiento que ellos originan (Sheard y Kakabadse, 2004, citado en Vázquez, 2013). 
El ejercicio del liderazgo es comparado a las destrezas que hay que poner en funcionamiento a la hora de circular a través de una calle. Branson (2010, citado en Gento y Cortés 2011:109) explica:

\begin{abstract}
Aunque el conductor tenga las destrezas necesarias para conducir el coche y sepa el destino final al que quiere llegar, lo que ocurre desde que comienza la conducción hasta que llega a su destino escapa totalmente a su control: debe ajustar su conducción a otros coches aparcados, a los coches que giran, a los que van despacio, a los que van muy rápido, a los que cruzan por la izquierda o por la derecha, a los peatones que cruzan por delante, a las posibles señales de giro, a los semáforos e, incluso al tiempo atmosférico (puesto que con lluvia debe conducirse más despacio). Simplemente, la conducción, en este caso, está influenciada por las acciones de otras personas, por la interpretación y juicio de lo que otros pueden hacer y por otros elementos objetivos o subjetivos que pueden ser estimados como relevantes para la conducción segura en un determinado entorno.
\end{abstract}

Si continuamos con el símil de conducir competentemente, el Plan Canario de Formación del Profesorado del bienio escolar 2013-2015 elabora ese manual de conducción, estableciendo la línea de actuación específica dirigida a la formación de los equipos directivos, marcando unos objetivos generales centrados en elevar la calidad del servicio educativo, profundizando en las técnicas y en los factores clave relacionados con el cumplimiento de las distintas funciones de la dirección, así como, conocer las herramientas necesarias para aprender a liderar, motivar, gestionar y dirigir un equipo de trabajo, todo ello realizando un análisis y evaluación del marco normativo y las directrices administrativas implicadas. En esta línea, dicho plan contempla los seminarios zonales de equipos directivos y servicios de apoyo a la escuela a través de la Red de Centros del Profesorado que con un carácter mensual se reúne para conducir, como dice Branson (2010), contemplando las diversas variables del camino y las acciones de los otros conductores con los que comparte trayecto e interactúa.

\title{
2.6. Formación del profesorado novel
}

Hemos ido reflexionando sobre la formación del docente con experiencia, pero ¿qué es del profesor novel que se enfrenta a los miedos e inseguridades propios de todo comienzo? Se trata de una etapa en la que la dimensión eficiencia juega un papel psicológico importante (Marcelo, 2009:14), donde las preocupaciones provienen principalmente por los cómo (cómo gestionar el grupo, cómo organizar el currículo, cómo evaluar,...), más que los porqué y cuándo. 
Simon Veenman (1984, en Marcelo, 2002) nos habla del concepto choque con la realidad para referirse al primer año de ejercicio. No se nos escapa que las necesidades y preocupaciones de estos docentes noveles requieren de apoyo técnico y administrativo, apoyo psicológico, feedback sobre mejoría en la práctica pedagógica y factores personales, además del apoyo emocional (Veeman, 1984 y Marcelo, 1993 citado en Marcelo, 2002).

Cooke y Pang (1991 citados en Marcelo, 2002) respecto a las necesidades de apoyo de los profesores principiantes, mencionan: la disciplina, la enseñanza (métodos y recursos), la motivación de los alumnos, la gestión del aula y el trabajo con la diversidad del alumnado. Creemos que atender a estas necesidades y cómo hacerlo, debe estar contemplado en toda oferta formativa inicial al docente, con el apoyo tutorizado del profesorado y abrigándolo desde la dirección del centro.

\subsection{Formación que permita afrontar retos}

Las evidencias sobre la complejidad de enseñar y aprender en el contexto actual refuerzan la idea de que la formación permanente es un apoyo imprescindible para afrontar los retos que el propio profesorado se formula en el día a día de su práctica docente (Montero, 2009 citado en Mediavilla, 2012).

Retos que exigen al profesorado, poner en marcha la maquinaria de sus conocimientos y destrezas, en cooperación con sus compañeros. Retos que pasan por reinventar la escuela, por innovar en nuevas formas de organización, por poner en práctica metodologías activas, por conocer las funcionalidades de los recursos tecnológicos, por aprender desde el propio currículo educativo, por tener muy claros los aprendizajes imprescindibles que debemos desarrollar en nuestro alumnado y poner la mirada en el cómo conseguirlo, para lograr una escuela inclusiva, cálida en afectos y de calidad, en un marco de convivencia positiva de todos sus miembros.

La consecución de estos retos pasa por admitir que la formación del profesorado debe ser un proceso continuo, de formación en acción, sistemático y organizado y entender que esta abarca toda la carrera docente (Marcelo y Vaillant, 2001 citado en Mediavilla, 2012) y pasa por admitir que el profesorado no puede estar o sentirse solo en el camino que emprende, que debe encontrarse acompañado y asistido por los servicios de apoyo a la escuela y la administración educativa en general.

\section{GRAdo DE SATISFACCIÓN DOCENTE}

Todo este camino de formación permanente debe llevar aparejado la satisfacción profesional como resultado de los logros alcanzados. La satisfacción general podríamos 
entenderla, tal como la han definido, como un estado espiritualmente gratificante (Sáenz y Lorenzo, 1993 citado en Caballero, 2002) o como defiende Brüggemann (1995, citado en Caballero, 2002), el resultado de una comparación entre lo que hace y lo que debería de hacer: a medida que la distancia disminuye, la satisfacción aumenta.

Tomando como variables el estado espiritualmente gratificante y el acortar el camino entre lo que el docente hace y debe hacer, la formación debe contemplar en su diseño, contenidos que colaboren con estas variables. Un diseño que le permita al docente, poner en práctica lo aprendido, viendo los resultados en su alumnado y en su interacción con el entorno laboral que comparte con otros colegas y las familias. Esa satisfacción se multiplica en beneficios porque con ella se consigue excelentes climas de trabajo.

\section{ConClusión}

Nuestro sistema educativo tiene ante sí el desafío ineludible de la mejora educativa y los cambios que esta conlleva. Este desafío tiene en el fortalecimiento de la profesión docente uno de sus componentes nucleares. Ese fortalecimiento exige una formación docente pluridimensional útil, que le permita la adquisición de competencias profesionales, de un modo sistemático y efectivo, para el desarrollo de una auténtica identidad profesional. El proceso de identidad profesional iría entre el reconocimiento de su propia identidad personal como profesional y la construcción de un plan de identidad profesional relacionado con los saberes específicos de su profesión y sus prácticas pedagógicas (Latorre, 2000 citado en Santibañez, 2012).

Una formación que, empíricamente fundada, contemple dónde se quiere llegar. Una formación que se evalúe para verificar si genera cambios y transformaciones reales en el sujeto y qué influencia tienen en su práctica docente (Fullan, 1991 citado en Medina, Jarauta y Urquizu, 2005).

Es evidente que el proceso educativo exige la conjugación de variables como docentes, educandos, familia, autoridades educativas, infraestructura física, estructura institucional, programas curriculares, pedagogías, materiales educativos y financiación. Y, por supuesto, no se puede delegar el peso de esta responsabilidad solo en el profesorado, pero como la actividad docente es una de esas variables relevante, la formación, que hemos analizado a lo largo de estas líneas, le empodera en su contribución a la calidad educativa. 


\section{Bibliografía}

CABALLERO, K. (2002). "El concepto de satisfacción en el trabajo y su proyección en la enseñanza". Profesorado, revista de currículum y formación del profesorado, 6 (1-2), 2002. Disponible en: http://www.ugr.es/ recfpro/rev61COL5.pdf

ESCUDERO, J. M. (2009b). "Comunidades docentes de aprendizaje, formación del profesorado y mejora de la educación”. Ágora, 10, pp. 7-31.

EURYDICE (2006): Quality Assurance in Teacher Education in Europe (Bruselas,

Unidad Europea de Eurydice) pp. 95.

GARCÍA, R. y CASTRO, A. (2012). La formación permanente del profesorado basada en competencias. Estudio exploratorio de la percepción del profesorado de Educación Infantil y Primaria. Universidad de Cantabria: Educatio Siglo XXI, Vol. 30 n $^{\circ} 1$, pp. 297-322.

GENTO, S. y CORTÉS, J. (2011). "Liderazgo para el Cambio Educativo”. I Congreso Internacional RIAICES, 22-24 de febrero de 2011.Universidade do Algarve Faro, Portugal.

GENTO, S. (2002). Instituciones Educativas para la Calidad Total. Madrid: La Muralla (3 ed.).

GRAU, S., GÓMEZ, C. y PERANDONES, T. (2009). La formación del profesorado como factor decisivo de la excelencia educativa. Disponible en: http://rua.ua.es/dspace/bitstream/10045/13199/1/PROPUESTAS\%20CAP.\%201.pdf

GÓMEZ, V. (2011). Desarrollo profesional del maestro. La competencia reflexiva. Evaluación de un programa formativo en el contexto panameño. Tesis doctoral. Universidad de Lleida.

GONZÁLES, G. (2013). Evolución de la identidad profesional de un docente novel de educación física: análisis a partir de la reflexión de sus experiencias personales y de su propia práctica. Tesis doctoral. Universidad de Valladolid.

MARCELO, C. (2002). La formación inicial y permanente de los educadores. Disponible en: http:// www. redes-cepalcala.org/inspector/DOCUMENTOS\%20Y\%20LIBROS/FORMACION/FORMACION\%20INICIAL\%20Y\%20PERMANENTE.pdf

MEDIAVILLA, D. (2012). El maestro novel. El papel de la formación inicial y continua en la construcción profesional. TFG. Universidad de Valladolid.

MEDINA, J. L., JARAUTA, B. y URQUIZU, C. (2005). "Evaluación del impacto de la formación del profesorado universitario novel: un estudio cualitativo". Revista de Investigación Educativa, Vol. 23, n 1, pp. 205-238.

Memoria del plan canario de formación del profesorado curso 2013-2014 (2015). Disponible en: http:// www3.gobiernodecanarias.org/medusa/campus/doc/doc/memorias/2013-14/secciones/

Resolución de la Dirección General de Ordenación, Innovación y Promoción Educativa por la que se relacionan los centros seleccionados para participar en la Red de Centros Innovadores para la Continuidad Escolar durante el curso 2014-2015.

RUPÉREZ, F. (2013). "Profesión y formación del profesorado de educación secundaria". Congreso Internacional Euro-Iberoamericano. Madrid, 16-19 de julio de 2013.

SANTIBAÑEZ, E. (2012). Revista Perspectiva Educacional, Vol. 51, n 1, p.1

TEJADA, J. (2001). “Función docente y formación para la innovación”. Revista de la Academia Mexicana de Educación, $\mathrm{N}^{\circ} 4$. 
TIANA, A. (2011). "Políticas de formación del profesorado y mejora de los sistemas educativos: algunas reflexiones a partir de la experiencia española". Revista Fuentes, 11; pp. 13-27.

VÁZQUEZ, A. (2013). "Interdependencia entre el liderazgo transformacional, cultura organizacional y cambio educativo: una reflexión". Revista Iberoamericana sobre Calidad, Eficacia y Cambio en Educación. Volumen 11, Número 1.

direccion.ceplaspalmas@gmail.com 